Document downloaded from:

http://hdl.handle.net/10251/142518

This paper must be cited as:

Yamanaka, E.; Tortajada-Genaro, LA.; Pastor Navarro, N.; Maquieira Catala, A. (06-2). Polymorphism genotyping based on loop-mediated isothermal amplification and smartphone detection. Biosensors and Bioelectronics. 109:177-183.

https://doi.org/10.1016/j.bios.2018.03.008

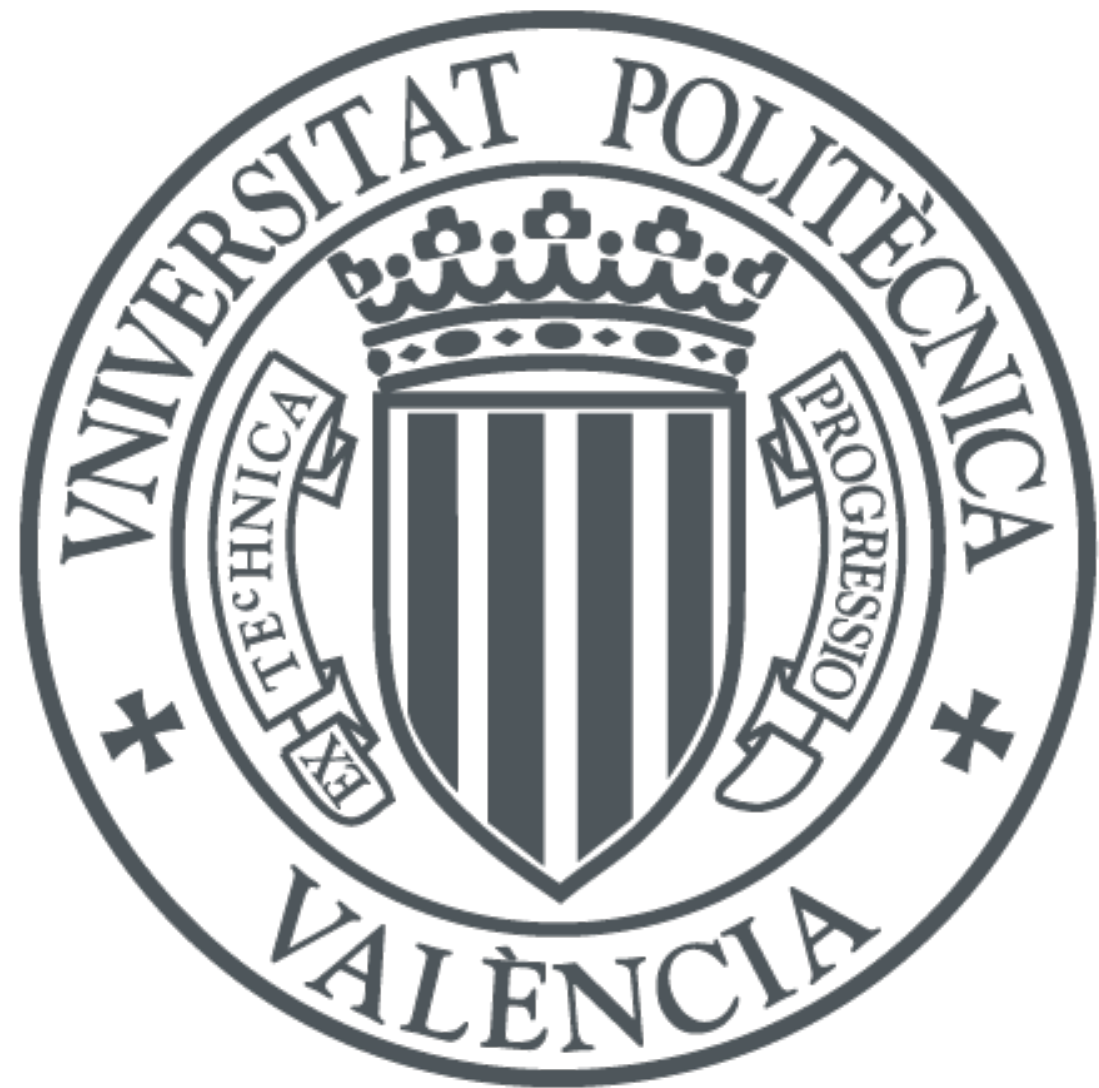

The final publication is available at

https://doi.org/10.1016/j.bios.2018.03.008

Copyright Elsevier

Additional Information 


\title{
Polymorphism genotyping based on loop-mediated isothermal amplification and smartphone detection
}

\author{
Eric Seiti Yamanaka ${ }^{(a)}$, Luis A. Tortajada-Genaro ${ }^{(\mathrm{a}, \mathrm{b}, \mathrm{c})}$, Nuria Pastor ${ }^{(\mathrm{a}, \mathrm{b})}$, Ángel \\ Maquieira $^{(\mathrm{a}, \mathrm{b}, \mathrm{c})}$
}

(a) Instituto Interuniversitario de Investigación de Reconocimiento Molecular y Desarrollo Tecnológico (IDM), Universitat Politècnica de València-Universitat de València, Spain

(b) Departamento de Química, Universitat Politècnica de València, Camino de Vera s/n, E46022 Valencia, Spain

(c) Unidad Mixta UPV-La Fe, Nanomedicine and Sensors, IIS La Fe, Valencia, Spain

\section{ABSTRACT}

The genotyping of a single-nucleotide polymorphism (SNP) is addressed through methods based on loop-mediated isothermal amplification (LAMP) combined with user-friendly optical read-outs to cover the current demand for point-of-care DNA biomarker detection. The modification of primer design and reaction composition improved the assay selectivity yielding allele-specific results and reducing false-positive frequency. Furthermore, the reduced cost, ease of use and effectiveness of colorimetric detection (solution and hybridization chip formats) were availed for the image capture by a smartphone, reching high sensitivity. In order to evaluate their discriminating capacities, LAMP-based methods were applied to human samples to genotype an SNP biomarker (rs1954787) located in the GRIK4 gene and related to the treatment response to anti-depressants drugs. Sensitive (limit of detection: 100 genomic DNA copies), reproducible ( $<15 \%$ error), fast (around $70 \mathrm{~min}$ ) and low-cost assays were accomplished. Patient subgroups were correctly discriminated, agreeing with reference sequencing techniques. The achieved analytical performances using the developed amplification-detection principles confirmed the approach potential for point-of-care optical DNA testing.

\section{Keywords}

Single-nucleotide polymorphism; loop-mediated isothermal amplification; point-of-care optical testing; smartphone; pharmacogenomics. 
Rapid advances made in DNA biomarkers research are providing us with a better understanding of disease mechanisms and drug action, which can lead to offering new personalised medicine opportunities (Wooley et al., 2014). The key step for implementing such systems in clinical routine is to employ highly efficient testing methods, which have to be accurate and sensitive enough to detect even minority variants, but also practical and economically feasible. In recent years, several studies have examined the capabilities of pointof-care (POC) genetic testing (Dobson et al., 2007). These tests generally include a costeffective field-portable device, along with an accurate, sensitive and simple DNA assay.

Amplification reactions are central to DNA-based diagnostic methods because sensitivity and selectivity depend on the effective increment in the copy number for the target region (Vashist et al., 2015). The most widely used amplification method is polymerase chain reaction (PCR), but it has some limitations for POC applications: a specific instrument for strict temperature control, susceptibility to amplification yield variations related to reaction conditions or the formation of air bubbles in miniaturised devices (Deng et al., 2015). Scientific advances have led to several enzymatic reactions run at constant temperature that can be used as an alternative to PCR-based amplification. Some recent reviews summarise isothermal amplification reactions and their use as analytical tools (Craw et al., 2012; Yan et al., 2014; Li 2015).

Loop-mediated isothermal amplification (LAMP), developed by Notomi et al. 2000, is the most extensively studied isothermal amplification technique. The main advantages over other approaches are its high amplification yield, good tolerance to inhibitors, short time and compatibility with several detection principles. The conventional approach relies on four primers to recognise six different sequences of the target DNA, which also leads to very high specificity. The action of a highly strand-displacing DNA polymerase (Bst polymerase) generates large amounts of dumbbell-like structures under isothermal conditions $\left(60-65^{\circ} \mathrm{C}\right)$. In virtue of these features, LAMP-based methods have been extensively applied to diagnose infectious diseases by detecting bacteria, viruses and parasites (Parida et al., 2008; Connelly et al., 2015).

In the last few years, several studies have demonstrated LAMP's capability to discriminate single-base variations, such as single nucleotide polymorphisms (SNPs) and somatic point detection. These methods are based on allele-specific hybridisation (Jiang et al., 2015,

64 Nakamura et al., 2007), and amplification using allele-specific primers (Zhang et al., 2014; 65 Yongkiettrakul et al., 2017) or a blocking agent (Itonaga et al., 2016). However, these methods 
usually rely on naked-eye visualisation or carry out the detection with expensive and bulky laboratory equipment (e.g. electrochemical stations, real-time turbidimeter or fluorometer). With the adequate integration to user-friendly detection technologies, these LAMP variants are appealing to develop POC testing. Examples of candidate clinical challenges are to select the correct oncological treatment with monoclonal antibodies (Shackelford et al., 2012), and to adjust drug doses in neuropathies and psychiatric disorders (Chan et al., 2011, Hamilton, 2015).

We herein explored these discrimination principles to develop high-performance POC systems. The first method involved the allele-specific oligonucleotide hybridisation of the LAMP products in the stem-loop region (LAMP-ASO). The second was based on the annealing selectivity of allele-specific inner primers (3'AS-LAMP), while discrimination in the third approach relied on DNA synthesis from a dumbbell-like starting structure (5' AS-LAMP). The key conditions to obtain adequate amplification yield, improve the discrimination factor and reduce false-positive frequency, were investigated. To this end, modifications in the primer/probe design, and variations in the amplification or hybridisation mix composition, were included.

Detection of allele specific products in POC scenarios also requires alternative detectors to previous LAMP approaches. In line with this, the features of consumer electronic devices are excellent as they are ubiquitous, low-cost, compact and high-performance products that can benefit advanced analytical measurements (Kido et al., 2000; Maquieira, 2012; Ozcan, 2014; Quesada-González et al., 2016). The sensing devices described for diagnostic purposes include compact disc drivers (Morais et al., 2014), flatbed scanners (Tortajada-Genaro et al., 2016) and mobile phones (Roda et al., 2016; Kanchi et al., 2018), among others. In this study, we explored the colorimetric detection of the developed homogeneous and heterogeneous LAMP assays supported by smartphone technology due to its widespread presence, portability and capacity to transmit data at a user-friendly interface. This integrated system also fulfils WHO requirements, and corresponds to the acronym “ASSURED”: affordable, sensitive, specific, user-friendly, rapid and robust, equipment-free, and delivered to those who need it.

\section{Material and Methods}

\subsection{Primers and probes}

LAMP primers and probes were designed for the target SNP according to the thermodynamic parameters described in the literature (Notomi et al., 2000, Tortajada-Genaro et al., 2017). The complete design strategy and oligonucleotide sequences can be found in the 
Supplementary Material. All the oligonucleotides used in this study were purchased from Eurofins (Luxembourg).

2.2. LAMP combined with allele-selective oligonucleotide hybridisation: LAMP-ASO method

In this approach, isothermal amplification was followed by hybridisation to the specific probes immobilised on planar polycarbonate chips $(25 \times 75 \mathrm{~mm})$. Non-allele selective LAMP amplification was carried out in $200 \mu \mathrm{L}$ propylene phials with primers that enclosed the polymorphic site. Each reaction $(12.5 \mu \mathrm{L})$ was composed of $1 \times$ isothermal amplification buffer (20 mM Tris-HCl, 10 mM (NH$)_{2} \mathrm{SO}_{4}, 50 \mathrm{mM} \mathrm{KCl,} 2$ mM MgSO $4,0.1 \%$ Tween 20, pH 8.8), 1.5 $\mathrm{M}$ betaine, further $6 \mathrm{mM} \mathrm{MgSO}_{4}, 1.2 \mathrm{mM}$ dNTPs, $10 \mu \mathrm{M}$ digoxigenin-11-deoxyuridine triphosphate (DIG-dUTP), $0.2 \mu \mathrm{M}$ of outer primers, $1.2 \mu \mathrm{M}$ of inner primers, $0.32 \mathrm{U} / \mu \mathrm{L}$ Bst polymerase 2.0 (New England Biolabs, USA) and $0.32 \mathrm{ng} / \mu \mathrm{L}$ (approximately 100 copies per $\mu \mathrm{L}$ ) of the studied DNA. Vials were incubated at $62^{\circ} \mathrm{C}$ for $60 \mathrm{~min}$ (digital heat block, VWR). Amplification products were then hybridised with the allele-specific oligonucleotide probes immobilised on chips in a microarray format. Probe arraying, hybridisation and colorimetric staining were performed according to the protocol developed in previous works (TortajadaGenaro et al., 2016, Yamanaka et al., 2017). The resulting hybridisation products anchored to the surface were recognised by horseradish peroxidase-conjugated antibodies and stained by 3,3',5,5'-tetramethylbenzidine to produce a precipitate.

A digital imaging technique was used to record the LAMP-ASO results. Array images were captured by a smartphone (MotoG first generation, Motorola) using a homemade capture chamber $(8.0 \times 6.7 \times 4.4 \mathrm{~cm})$. This chamber had a frontal rectangular aperture for the smartphone camera, a lateral hole to illuminate the array by an external optical fibre light source (20W power, 3,000 K colour temperature, LE.5209 model, Euromex, Holland), and an inferior aperture to insert the assay chip. The image was captured after adjusting both focus and exposure (75\% saturation) and was converted into a tagged image file format on a 16-bit (065,535) greyscale with the ImageJ software (National Institutes of Health, USA). Images were analysed and the resulting spot intensities were expressed in signal-to-noise ratio terms.

\subsection{Allele-specific LAMP: 3'AS-LAMP and 5' AS-LAMP formats}

Two homogeneous amplification formats were assayed using allele-specific primers (see Supplementary Material). In each case, discrimination was achieved using two reaction mixtures to amplify the wild-type variant (wild-type primers) or the mutant variant (mutant 
primers). For 3' AS-LAMP format, the polymorphism was located at the 3'-end of the forward inner primer (FIP), leading two allele-specific primers and a reverse inner primer common to both reaction mixtures. Therefore, the reaction mixture composition varied from the previously described non-selective LAMP by using each FIP primer, 1.25 M betaine and $300 \mu \mathrm{M}$ hydroxynaphtol blue. In the 5' AS-LAMP format, the polymorphism was located at the 5'-end of both FIP and BIP, and the difference in mixture composition was the betaine and dyer concentrations, which were $0.75 \mathrm{M}$ and $300 \mu \mathrm{M}$, respectively. On-chip amplification was carried out with a rhombic chamber chip (reaction volume $10 \mu \mathrm{L}$, Zeonor material) supplied by microfluidic ChipShop (Germany). Inlets and outlets were connected directly to Tygon tubing. Chips were loaded with the amplification samples and were incubated at $62^{\circ} \mathrm{C}$ for 60 $\min$.

Smartphone imaging enabled end-point direct colorimetric detection. For this purpose, the reaction chip with a reference colour palette was placed in the previously described detection assembly. The AssayColor software (Alidans, Italy), installed in the smartphone, was used to capture and analyse images. This scientific application, developed for the Android operating system, provided colour intensities in the red, green and blue channels (RGB) for each LAMP product. The R/G intensity ratio was selected as an analytical signal. were obtained from the buccal smear samples with the Purelink Genomic DNA mini kit (Thermo Fisher Scientific, USA). Purified products were eluted with Tris-HCl buffer (Tris 10 $\mathrm{mM}, \mathrm{pH}$ 8.6) and their genomic DNA content was quantified in a NanoDrop 2000 spectrophotometer (Thermo Fisher Scientific, USA). A 260/280 nm absorbance ratio above 1.8 was considered to determine adequate purity. Extracts were diluted to $4 \mathrm{ng} / \mu \mathrm{L}$ and stored at $20^{\circ} \mathrm{C}$ until further use. Subsequently, samples were submitted to the LAMP-ASO, 3'AS-LAMP and 5'AS-LAMP methods. A no-template control and a Salmonella typhimurium DNA extract were used to check for false-positive assays. A discrimination index was calculated from the signal of the wild-type (WT) and mutant (MUT) responses according to the following equation: (WT - MUT)/(WT + MUT). The genotype was assigned according to discrimination 


\subsection{Genotyping validation}

Two techniques were used to confirm patients’ genotypes: Sanger sequencing and allelespecific PCR.

For Sanger sequencing, each PCR reaction was carried out in a mixture $(12.5 \mu \mathrm{L})$ that contained 1x amplification buffer, $3 \mathrm{mM} \mathrm{MgCl}_{2}, 200 \mu \mathrm{M}$ dNTPs, $300 \mathrm{nM}$ of the forward and reverse primers, 0.5 units of Taq polymerase (Biotools, Spain) and 20 ng of genomic DNA per reaction. Amplification was carried out in a UnoCycler thermal cycler (VWR, USA) according to the following programme: initial denaturation at $95^{\circ} \mathrm{C}$ for $5 \mathrm{~min}$, followed by 35 amplification cycles of denaturation $\left(95^{\circ} \mathrm{C}\right.$ for $\left.30 \mathrm{~s}\right)$, annealing $\left(60^{\circ} \mathrm{C}\right.$ for $\left.30 \mathrm{~s}\right)$ and elongation $\left(72^{\circ} \mathrm{C}\right.$ for $30 \mathrm{~s}$ ), and a final extension step at $72^{\circ} \mathrm{C}$ for $5 \mathrm{~min}$. The resulting amplification products were diluted, extended with fluorescent dideoxynucleotides (Big Dye Terminator Cycle Sequencing Kit v3.1, Thermo Fisher Scientific, USA), and analysed in a fluorescencecapillary sequencer (ABI 3130xl Genetic Analyzer, Applied Biosystems, USA).

Allele-specific PCR (AS-PCR) was based on the use of two forward primers that differed at the 3'-end nucleotide, and were complementary to the wild-type or mutant variant. An additional mismatch at the penultimate nucleotide was included. The amplification conditions were identical to those previously described for PCR, except for the use of the allele-specific primers and an annealing temperature of $62^{\circ} \mathrm{C}$. End-point fluorescence was measured to confirm amplification. Products were diluted in 0.5× SYBR Safe (Invitrogen, USA) on a 96well black polystyrene plate and analysed in a plate reader (Victor 3TM V1420, Perking Elmer, Finland) at excitation and emission wavelengths of 485 and 535 nm, respectively.

The Statgraphics Centurion statistical package for Windows v.16 was used for the data analysis. 


\subsection{ASO-LAMP set-up}

SNP discrimination was performed with the combination of isothermal DNA amplification and hybridisation with allele-selective probes in a solid-phase format.

The first step was the oligonucleotide design (primers and probes). There were two design options according to the target polymorphism location in the LAMP product loopstructure: central position (double-strand region) or loops (single-strand regions). The second option was chosen to improve the hybridisation yield to the array probe (Fig. 1a). A thermodynamic analysis was used to select the candidate probes that maximised the hybridisation of perfect-match pairs (wild-type or mutant) and hampered the coupling of mismatched products (wild-type product/mutant probe or mutant product/wild-type probe). An additional design restriction was the central position of the polymorphic mismatch in the probe to increase assay selectivity. The selected sequences produced wide variation in standard free energies, expressed as the difference between the single-base mismatch ( $\Delta \mathrm{G}^{\mathrm{o}}$ mutant) and the perfect match ( $\Delta \mathrm{G}^{\mathrm{o}}$ wild-type). Estimated values were 3.3-4.5 kcal $/ \mathrm{mol}$.

The LAMP reaction was optimised to selectively amplify the targeted region using the designed non-allele-specific primers. Reagent concentrations (enzyme, inner primers and outer primers), amplification temperature and reaction time were studied by the fluorescence analysis (see the Supplementary Material). Negative controls (non-human DNA) produced a signal comparable to the background, while the amplification of the human DNA template generated a significantly distinguishable signal (Fig. 1b). The wild-type and mutant templates produced similar amplification curves, and the time selected for the end-point analysis was $60 \mathrm{~min}$. The amplification factor was $(2.6 \pm 0.8) \times 10^{8}$, which gave a 23 -fold higher yield than a typical PCR using the same external primers and the amount of the initial DNA template.

The next experiments focused on the selective hybridisation to the probes anchored to the plastic chip, by directly dispensing the end-point LAMP product on the probe arrays. This

214 approach is simpler and more efficient than combining PCR-based methods with microarray detection because an intermediate (thermal or chemical) denaturalisation step is generally

216 required (Wooley et al., 2014, Tortajada-Genaro et al., 2016). The probe immobilisation 217 parameters (concentration, drop volume, and surface treatment) and the hybridisation variables 218 (buffer composition, time and washing cycle) were studied to balance yield and selectivity, as 219 described in the Supplementary Material. The most critical variable to achieve selective 220 hybridisation was buffer composition, particularly formamide concentration (Figure 1c). Under 
the optimal conditions (1x sodium saline citrate buffer, $30 \%$ formamide), a detectable signal was obtained for the perfect-matched duplexes (wild-type and mutant homoduplex), while a background-equivalent response was acquired for the mismatch hybrids. matched primer-template pairs. The LAMP assays generated false-positives due to the formation of unexpected primer structures, as observed in other studies (Connelly et al., 2015; Wang et al., 2015). Therefore, several modifications were made to increase assay selectivity in the AS-LAMP formats. Firstly, an additional mismatch was deliberately added to the penultimate nucleotide of the allele-specific primers. Major destabilisation of the hybridization process was estimated for the mismatch probes, where the calculated variation of the standard free energies was about 1.2 - $2.8 \mathrm{kcal} / \mathrm{mol}$. Secondly, the effect adding betaine to the amplification mix was evaluated. This amino acid analogue is often used for destabilising dsDNA and for reducing the sequence composition influence on the melting temperature. The experiments showed that adding betaine eliminated the false-positive results associated with the mismatch hybrids (Fig. 2). However, increasing the betaine concentrations also led to the undesired inhibition of the perfect-matched duplexes. The inhibition effect was more prominent in the 5' allele-specific format than in the 3' one. This could be explained by the lesser stability of the associated perfect-match hybrids (about $5 \mathrm{kcal} / \mathrm{mol}$ ) and a different number of allele-specific primers (two in the 5' format and one in the 3' format). In summary, the results at the selected values (1.25 M for 3'AS-LAMP and 0.75 M for 5'AS-LAMP) showed better amplification selectivity compared to conventional conditions.

Amplification kinetics was studied to verify the discrimination capacity and the assay turnout time for the LAMP reactions. Both the allele-specific methods showed adequate selectivity as the real-time signals for the no-template control and the non-human DNA extract (Salmonella culture) were similar to the background. When the perfectly matched primers were used, amplification started at $40 \mathrm{~min}$ for the 3' and 5' allele-specific approaches, while the mismatched primers generated a signal after a delay that went beyond $30 \mathrm{~min}$ in both cases. It is worth noting that the stability difference between the previously described matched and mismatched duplexes was also reflected in the amplification kinetic profiles. Longer delays for the mismatched pairs were found in the 3' format. After considering the results, a 60-minute amplification time was selected for the following experiments to prevent the formation of nonspecific products during the assay. 


\subsection{Smartphone detection}

257

The detection of the previously described allele-selective products was achieved with conventional laboratory instruments; i.e. fluorescence qPCR thermocycler, fluorescence spectrophotometer or fluorescence scanner. The next challenge was to adapt the methods for colorimetric detection using a smartphone (complementary metal-oxide-semiconductor, CMOS sensor) suitable for point-of-care testing. An integrated detection device was assembled for chip reading, which comprised a light source, a dark chamber and the smartphone aligned to the chip (Fig. 3). To guarantee inter-assay measurement robustness, a colour pattern (a violet to blue scale) was photographed together with the assay platforms. The specific measuring conditions were optimised to digitalise the array profile by the smartphone camera, as the Supplementary Material describes. Image resolution, expressed as pixel width, was $17 \mu \mathrm{m}$.

For the LAMP-ASO approach, a colorimetric detection method for the probe-LAMP product hybrids based on an immunorecognition step (digoxigenin/primary antibody/secondary antibody system) and enzymatic staining (horseradish peroxidase/colorimetric substrate system) was studied. If hybridisation was positive, a blue precipitate was generated on the spot by attenuating the captured optical density (reflectionmode detection). The intensity of each array spot (400 $\mu \mathrm{m}$ diameter) was calculated as the average of 448 pixels. A perfect-match interaction (LAMP product/probe) produced signals up to 56,000 a.u. in 16-bit greyscale units, while the chip background values were in the range of $7,000 \pm 400$ a.u.. Therefore, the spot intensities discriminated positive and negative recognition events depending on the probe/product pair. Statistical significance was calculated by a Student's $t$-test, and p-values were $<0.05$ in all cases. This study demonstrates, for the first time, the colorimetric detection of allele-specific hybridisation LAMP products, which produces excellent versatility and is a key factor to make a simpler reader-suitable method for POC applications.

For the AS-LAMP approaches, the addition of a magnesium indicator (hydroxynaphtol blue) was evaluated (Zhang et al., 2014b). Along with the capacity of the isothermally amplifying double strand DNA, a very high yield is an interesting advantage that LAMP offers over conventional PCR and other isothermal amplification methods, as it allows a subsequent direct colorimetric detection with a smartphone. This staining method was simple and did not require any additional devices (i.e. ultraviolet source, wavelength filters, magnification lens). Detection was achieved with no post-amplification steps. To improve the recorded responses 
(scattered light), the concentration of hydroxynaphtol blue was gradually increased and the light intensity for the RGB channels was recorded (Fig. 3). Concentrations above $300 \mu \mathrm{M}$ provided a significant signal of red channel for the positive amplifications compared to the negative controls (test $\mathrm{t}: \mathrm{t}=2.25, \mathrm{p}<0.05$ ). From the obtained results, the proposed modification of ASA-LAMP approaches showed excellent signal discrimination, which indicates its potential as a polymorphism biomarker analysis tool.

\subsection{Comparing methods}

The main features and analytical performances of the three methods were subsequently compared (Table 1). Analytical sensitivity and reproducibility were calculated from the consecutive dilutions of a genomic human DNA template. Although naked-eye colour observation was possible to visualise positive amplification (violet to sky blue), the use of an imaging/sensing device guaranteed reliable measurements when smaller amounts of the target SNP were present in the sample and colour change was subtle. The estimated limit of detection was 100 copies for the all LAMP-smartphones-based methods. Thus the required amount of genomic DNA was smaller than previous LAMP approaches (Nakamura et al., 2007, Itonaga et al., 2016), some genotyping assays (Gibriel et al., 2017) and sequencing techniques (Goodwin et al., 2016). Assay repeatability, calculated from replicates, yielded error rates under $15 \%$ in all the formats, which were lower than those obtained by naked eye visualisation and similar to other SNP methods that have been applied to human samples.

The technical requirements for developing point-of-care systems were also evaluated. The estimated reagent cost of LAMP-ASO was 2.65-fold higher than the AS variants, mainly because of immunoreagent prices. Compared with the corresponding PCR approaches, LAMP assays were more expensive (about 1.5-fold), mainly due to the cost of enzymes (Bst polymerase versus Taq polymerase). In contrast, the LAMP approaches only required a lowcost heating system $\left(62^{\circ} \mathrm{C}\right.$; i.e. heater) compared to the conventional thermal cycler used in

314 PCR-based methods, along with a cheaper and more practical detector. The LAMP methods 315 also worked in shorter analysis times than their equivalent PCR approaches. The AS-LAMP 316 formats were the quickest (70 $\mathrm{min})$ compared to LAMP-ASO (140 min), AS-PCR (120 min) or PCR-ASO (190 min), mostly because of the shorter amplification times in the LAMP-based

318 methods. Hence these results are similar, or better, than those obtained for previous LAMP approaches (Connelly et al., 2015; Safavieh et al., 2016; Itonaga et al., 2016). 
Psychiatric pharmacogenetics is a candidate field for developed POC genotyping methods (Milanesi et al., 2015). As proof of concept, the genotyping of the rs1954787 polymorphism, located in the GRIK4 gene, was selected to determine the genetic predisposition of antidepressant treatment from the human DNA $(n=15)$ extracted from buccal swabs. Only by following the developed methodology were signals sufficiently different to achieve a specific response profile depending on the genetic variant. Figure 4 shows the subsequent discrimination graph. The three methods provided the same genotypes for all patients, except for patient 8 in the LAMP-ASO approach. Nevertheless, the homogeneous approaches (3' ASLAMP and 5' AS-LAMP methods) provided clearer discrimination factors than the solid hybridisation format (LAMP-ASO) due to their lower signals for the mismatched reaction mixtures. Among the analysed samples, six patients (40\%) were identified as being mutant homozygous (CC) which can be related to a better chance of positive responses to depression treatment (Horstmann, 2010). There were also six heterozygous patients (40\%), who were expected to give a normal response for drugs like citalopram. Finally, the results indicated that three (20\%) subjects presented a homozygous wild-type genotype (TT), which indicates a higher risk of a non-response. Another comparison of the reference results (Sanger sequencing and AS-PCR) revealed a perfect correlation with the genotypes determined by the LAMPbased assays.

The clinical implications of this in vitro diagnostic assay were analysed. Major depressive disorder affects were about $10-15 \%$ of the population (annually), with a degree of uncertainty about the individual efficacy of the antidepressant treatment (Kawaguchi et al., 2014). The discrimination of specific polymorphisms can enable quick personalised patient management with a strong effect on therapy. Clinical trials have identified an association of rs1954787 with therapy effectiveness, and have reported that CC homozygotes are more likely to respond to treatment than TT homozygotes. Therefore, a simple low-cost genotyping tool can support the better dosing of antidepressants.

\section{Conclusion}

This research confirms the excellent features of LAMP as a viable alternative to current methodologies whose aim is genotyping purposes in order to overcome the associated technical barriers. This study particularly supports the oligonucleotide design and the selection of reaction conditions for colorimetric detection in both homogeneous and heterogeneous formats. An accurate control of these experimental variables is required because false-positive 
results are more frequent than for PCR-based methods. Our results endorse the technical capabilities of smartphones as analytical readers for molecular diagnostic systems. Despite having a worse optical resolution than benchtop instruments, CMOS sensor chips incorporated into phone cameras offer adequate imaging features and widespread availability, which make them ideal detectors for cost-effective assays. Compared to other electronic devices, smartphone technology has additional advantages, such as assay reader, given its capability to transmit data, ubiquity and users' familiarity to handle it.

The achieved LAMP discrimination process and low-cost detector combination shows excellent performance and a wide dynamic range, which allows the technique to be extrapolated other target genetic biomarkers. This offers researchers the chance to develop integrated systems, which enable quicker monitoring of genetic predispositions to develop certain diseases or to predict genomic-related responses to drug therapies.

\section{Acknowledgements}

The authors acknowledge the financial support received from the Generalitat Valenciana (Project GVA-PROMETEOII/2014/040 and GRISOLIA/2014/024 PhD grant) and from the Spanish Ministry of Economy and Competitiveness (MINECO CTQ2013-45875-R Project).

\section{REFERENCES}

Chan, A., Pirmohamed, M., \& Comabella, M., 2011, Ann. Neurol. 70, 684-697.

Connelly, J. T., Rolland, J. P. \& Whitesides, G. M., 2015. Anal. Chem. 87, 7595-7601.

Craw, P., \& Balachandran, W., 2012. Lab Chip. 12, 2469-2486.

Deng, H., \& Gao, Z., 2015. Anal. Chim. Acta. 853, 30-45.

Dobson, M. G., Galvin, P., \& Barton, D. E., 2007. Expert Rev. Mol. Diagn. 7, 359-370.

Gibriel, A. A., \& Adel, O., 2017. Mutat Res Rev Mutat Res. 773, 66-90.

Goodwin, S., McPherson, J. D., \& McCombie, W. R., 2016. Nat. Rev. Genet. 17, 333-351.

Hamilton, S. P., 2015. Biol. Psychiatry. 77, 29-35.

Horstmann, S., Lucae, S., Menke, A., Hennings, J. M., Ising, M., Roeske, D., Müller-Myhsok, B., Holsboer, F. \& Binder, E. B., 2010. Neuropsychopharmacology. 35, 727-740.

Itonaga, M., Matsuzaki, I., Warigaya, K., Tamura, T., Shimizu, Y., Fujimoto, M., Kojima, F., Ichinose, M. \& Murata, S. I., 2016. PloS one, 11, e0151654.

Jiang, Y. S., Bhadra, S., Li, B., Wu, Y. R., Milligan, J. N., \& Ellington, A. D., 2015. Anal. Chem. 87, 3314-3320.

Kanchi, S., Sabela, M. I., Mdluli, P. S., \& Bisetty, K., 2018. Biosens. Bioelectron. 102, 136149.

Kawaguchi, D. M., \& Glatt, S. J. 2014. Pharmacogenomics 15, 1451-1459.

Kido, H., Maquieira, A., \& Hammock, B. D., 2000. Anal. Chim. Acta. 411, 1-11.

Li, J., \& Macdonald, J., 2015. Biosens. Bioelectron. 64, 196-211. 
Maquieira, A. (2012). Compact discs technology for clinical analysis of drugs, in: Herold, K. E., Rasooly, A. (Eds.), Biosensors and molecular technologies for cancer diagnostics. CRC Press. Boca Raton, pp. 417-440

Milanesi, E., Bonvicini, C., Congiu, C., Bortolomasi, M., Gainelli, G., Gennarelli, M., \& Minelli, A., 2015. Genet. Res. 97, e14.

Morais, S., Tortajada-Genaro, L., \& Maquieira, Á., 2014. Expert Rev. Mol. Diagn. 14, 773775

Nakamura, N., Ito, K., Takahashi, M., Hashimoto, K., Kawamoto, M., Yamanaka, M., Atsuo Taniguchi, A., Kamatani, N. \& Gemma, N., 2007. Anal. Chem. 79, 9484-9493.

Notomi, T., Okayama, H., Masubuchi, H., Yonekawa, T., Watanabe, K., Amino, N., \& Hase, T., 2000. Nucleic Acids Res. 28, e63-e63.

Ozcan, A., 2014. Lab Chip 14, 3187-3194.

Parida, M., Sannarangaiah, S., Dash, P. K., Rao, P. V. L., \& Morita, K., 2008. Rev. Med. Virol. 18, 407-421.

Quesada-González, D., \& Merkoçi, A., 2017. Biosens. Bioelectron. 92, 549-562.

Roda, A., Michelini, E., Zangheri, M., Di Fusco, M., Calabria, D., \& Simoni, P., 2016. Trends Analyt Chem. 79, 317-325.

Safavieh, M., Kanakasabapathy, M. K., Tarlan, F., Ahmed, M. U., Zourob, M., Asghar, W., \& Shafiee, H., 2016. ACS Biomater Sci Eng. 2, 278-294

Shackelford, R. E., Whitling, N. A., McNab, P., Japa, S., \& Coppola, D., 2012. Genes Cancer. 3, 459-466.

Tortajada-Genaro, L. A., Mena, S., Niñoles, R., Puigmule, M., Viladevall, L., \& Maquieira, Á., 2016. Anal. Bioanal. Chem. 408, 2339-2345.

Tortajada-Genaro, L. A., Puchades, R., \& Maquieira, Á., 2017. J. Pharm. Biomed. Anal. 136, $14-21$.

Vashist, S. K., Luppa, P. B., Yeo, L. Y., Ozcan, A., \& Luong, J. H., 2015. Trends Biotechnol. 33, 692-705.

Wang, D. G., Brewster, J. D., Paul, M. \& Tomasula, P. M., 2015. Molecules 20, 6048-6059.

Woolley, C. F., \& Hayes, M. A., 2014. Emerging technologies for biomedical analysis. Analyst 139, 2277-2288.

Yamanaka, E. S., Tortajada-Genaro, L. A., \& Maquieira, Á., 2017. Microchim. Acta 184, 14531462.

Yan, L., Zhou, J., Zheng, Y., Gamson, A. S., Roembke, B. T., Nakayama, S., \& Sintim, H. O., 2014. Mol. Biosyst. 10, 970-1003.

Yongkiettrakul, S., Kampeera, J., Chareanchim, W., Rattanajak, R., Pornthanakasem, W., Kiatpathomchai, W., \& Kongkasuriyachai, D., 2017. Parasitol. Int. 66, 964-971.

Zhang, L., Zhang, Y., Wang, C., Feng, Q., Fan, F., Zhang, G., Kang, X., Qin, X., Sun, J., Li, Y. \& Jiang, X., 2014. Anal. Chem. 86, 10461-10466.

Zhang, F., Wang, R., Wang, L., Wu, J., \& Ying, Y., 2014. Chem. Commun. 50, 14382-14385. 